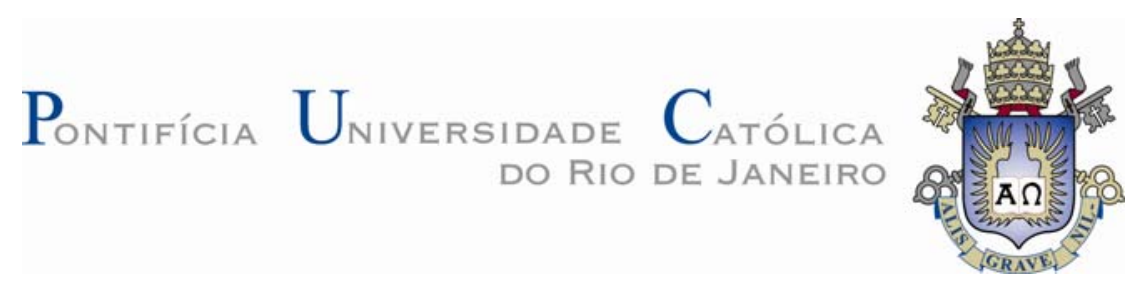
Gustavo Fernando Dorregaray Portilla

Simulação de Sistemas de Refrigeração em Supermercados

Dissertação apresentada ao Programa de PósGraduação em Engenharia Mecânica da PUC-Rio como requisito parcial para obtenção do título de Mestre em Engenharia Mecânica.

Orientador: José Alberto dos Reis Parise Co-orientador: Samuel Fortunato Yana Motta 


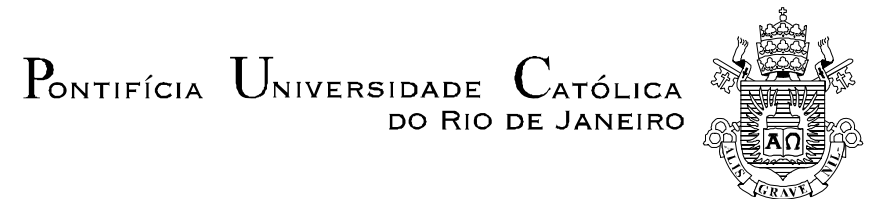

Gustavo Fernando Dorregaray Portilla

\title{
Simulação de sistemas de refrigeração em supermercados
}

\author{
Dissertação apresentada como requisito parcial para \\ obtenção do grau de Mestre pelo Programa de Pós- \\ Graduação em Engenharia Mecânica do Centro Técnico \\ Científico da PUC-Rio. Aprovada pela Comissão \\ Examinadora abaixo assinada.
}

Prof. José Alberto dos Reis Parise Orientador Departamento de Engenharia Mecânica - PUC-Rio

Prof. Carlos Eduardo Leme Nóbrega Centro Federal de Educação Tecnológica Celso Suckow da Fonseca - RJ

Prof. Sergio Leal Braga

Departamento de Engenharia Mecânica - PUC-Rio

Prof. Alcir de Faro Orlando

Departamento de Engenharia Mecânica - PUC-Rio

Prof. José Eugenio Leal

Coordenador Setorial do Centro Técnico Científico - PUC-Rio

Rio de Janeiro, 30 de abril de 2010 
Todos os direitos reservados. É proibida a reprodução total ou parcial do trabalho sem autorização da universidade, do autor e do orientador.

\section{Gustavo Fernando Dorregaray Portilla}

Graduou-se em Engenharia Mecânica na Faculdade de Ciências e Engenharia da PUCP (Pontifícia Universidade Católica do Peru), em 2006. Atualmente tem atuado na área de Petróleo e Energia, concentrando seus esforços na análise de consumo energético em supermercados e seu impacto ambiental das alternativas apresentadas.

Ficha Catalográfica

Portilla, Gustavo Fernando Dorregaray

Simulação de sistemas de refrigeração em supermercados / Gustavo Fernando Dorregaray Portilla ; orientadores: José Alberto dos Reis Parise, Samuel Fortunato Yana Motta. - 2010.

128 f.: il. ; $30 \mathrm{~cm}$

Dissertação (Mestrado)-Pontifícia Universidade Católica do Rio de Janeiro, Departamento de Engenharia Mecânica, Rio de Janeiro, 2010.

Inclui bibliografia

1. Engenharia mecânica - Teses. 2. Refrigeração. 3. Supermercados. 4. Energia. 5. Impacto ambiental. 6. Simulação. I. Parise, José Alberto dos Reis. II. Motta, Samuel Fortunato Yana IV. Pontifícia Universidade Católica do Rio de Janeiro. Departamento de Engenharia Mecânica. V. Título.

CDD: 621 


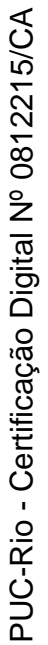

“A sorte ajuda os audazes” - Virgílio. 


\section{Agradecimentos}

A meus pais Fernando e Charo e meu irmão Eduardo por seu amor e apoio incondicional.

A José Alberto dos Reis Parise, meu orientador, pela amizade e enorme paciência que teve comigo durante estes anos.

A Samuel Fortunato Yana Motta, meu co-orientador, pelo conhecimento fornecido ao longo do desenvolvimento desta dissertação e pela motivação e confiança que teve comigo para levá-la a cabo.

A meus amigos de apartamento Raul, Ivan, Cesar e Harry, pela amizade e pelos bons momentos compartilhados nestes anos.

Agradecimentos em particular para os órgãos de fomento à pesquisa CNPq, CAPES e FAPERJ, pelo apoio financeiro fornecido, sem o qual este trabalho simplesmente não teria sido possível. Por outro lado, meu agradecimento é também dirigido à empresa Honeywell Inc., pelo apoio financeiro ao projeto. 


\section{Resumo}

Portilla, Gustavo Fernando Dorregaray; Parise, Jose Alberto dos Reis. Simulação de sistema de refrigeração em supermercados. Rio de Janeiro, 2010. 128p. Dissertação de Mestrado - Departamento de Engenharia Mecânica, Pontifícia Universidade Católica do Rio de Janeiro.

Desenvolveu-se um modelo termodinâmico para cálculo do consumo energético e do impacto ambiental (LCCP e TEWI) em sistemas de refrigeração de supermercados para três diferentes configurações (expansão direta, fluido secundário e em cascata). O modelo termodinâmico trabalha solidário com o pacote de cálculo de propriedades REFPROP e determina diversos parâmetros de funcionamento do ciclo de refrigeração, tais como o COP e a capacidade de refrigeração. O modelo simula sistemas operando com substâncias puras ou misturas delas, podendo estas ser azeotrópicas ou não-azeotrópicas. Procede-se a uma análise do tipo BIN (Intervalo) a partir de informações climatológicas anuais de cada cidade e ao cálculo do consumo anual de energia para cada intervalo considerando-se o consumo do compressor, das bombas, se for o caso específico da utilização de fluido secundário, e também o consumo energético dos expositores frigoríficos. Realizou-se, também, uma análise da carga total (inventário) do refrigerante no supermercado. Finalmente, conhecidos o consumo energético anual, a massa total de refrigerante contido no sistema e estimativa de seu vazamento anual, e outros fatores ambientais, procedeu-se ao cálculo do LCCP (Life cycle climate performance) e do TEWI (Total equivalent warming impact), em quilogramas equivalentes de $\mathrm{CO}_{2}$. Um programa computacional, com interface baseada na plataforma Visual-Basic 6.0, com aplicações para Excel, foi desenvolvido para a solução dos sistemas de equações resultantes do modelo matémático desenvolvido. Os resultados obtidos com o código computacional foram comparados com dados de campo e de outros modelos.

\section{Palavras-chave}

Refrigeração; supermercados; energia; impacto ambiental; simulação. 


\section{Abstract}

Portilla, Gustavo Fernando Dorregaray; Parise, Jose Alberto dos Reis (Advisor). Refrigeration Systems simulation in Supermarkets. Rio de Janeiro, 2010. 128p. MSc. Dissertation - Departamento de Engenharia Mecânica, Pontifícia Universidade Católica do Rio de Janeiro.

A thermodynamic model was developed for the evaluation of energy consumption and environmental impact (LCCP and TEWI) of supermarket refrigeration systems operating in three different configurations (direct expansion, secondary fluid and cascade). The model simulates refrigeration systems operating with pure substances or a mixture (azeotropic or non-azeotropic) of them. A BIN analysis is carried out taking into account year-round climate data for each city and the annual energy consumption, in each time interval, of each component: compressor, pumps, freezers and coolers. Refrigerant inventory was also calculated. Finally, with the annual energy consumption, total refrigerant charge, annual leakage estimate, environmental impact indicators LCCP (Life cycle climate performance) and TEWI (Total equivalent warming impact), in kilograms equivalent of $\mathrm{CO}_{2}$, were calculated. A computational code, with interface based on Visual Basic 6.0 platform, was developed for the solution of the resulting systems of equations that form the mathematical model. Predicted results were compared with field data and with data predicted from other models.

\section{Keywords}

Refrigeration, supermarkets, energy, environmental. 


\section{Sumário}

$\begin{array}{ll}\text { 1. Introdução } & 19\end{array}$

1.1. Consumo energético em supermercados 19

1.2. Principais características dos sistemas de refrigeração em supermercados 20

1.3. Aspecto ambiental - Indicadores 27

1.4. Objetivo do trabalho 31

1.5. Justificativa 31

1.6. Estado da arte 32

1.7. Contribuição do presente trabalho 35

1.8. Conteúdo do Trabalho 36

2. Modelo matemático 37

2.1. Descrição dos sistemas

2.1.1. Sistema de expansão direta $\quad 37$

2.1.2. Sistema com fluido secundário 40

2.1.3. Sistema de ciclos em cascata 43

2.2. Hipóteses simplificadoras para todos os sistemas 45

2.3. Calculo da carga térmica do supermercado 46

2.4. Queda de pressão nas tubulações 47

2.5. Modelo termodinâmico 50

2.5.1. Equações de conservação 51

2.5.2. Cálculo da vazão mássica 52

2.5.3. Eficiência isentrópica 54

2.5.4. Condensador 54

2.5.5. Evaporador 54

2.5.6. Sub-resfriador Mecânico 55

2.5.7. Outros elementos do ciclo 56

2.5.8. Calculo do COP (Coeficiente de desempenho) do ciclo termodinâmico 58

2.6. Cálculo da potência dos compressores 58 
2.6.1. Cálculo da potência dos compressores de baixa temperatura 58

2.6.2. Cálculo da potência dos compressores de temperatura média 58

2.7. Cálculo da potência elétrica das bombas (Sistema com fluido secundário) 59

2.7.1. Calculo da potencia elétrica das bombas de baixa temperatura 59

2.7.2. Cálculo da potência elétrica das bombas de média temperatura 60

2.8. Cálculo do consumo anual de energia dos compressores mediante a análise BIN

2.9. Cálculo do consumo anual de energia no circuito de baixa temperatura e média temperatura 62

2.10. Cálculo da massa total de refrigerante no supermercado 62

2.10.1. Cálculo da massa de refrigerantes nas tubulações 62

2.10.2. Cálculo da massa de refrigerante nos distribuidores de sucção, descarga e de líquido 64

2.10.3. Cálculo de massa de refrigerante no condensador 64

2.10.4. Cálculo de massa de refrigerante no evaporador 66

2.10.5. Cálculo da massa de refrigerante no tanque Reservatório de líquido

2.10.5.1. Tanques reservatórios verticais 67

2.10.5.2. Tanques reservatórios horizontais 68

2.10.6. Cálculo de massa de refrigerante no compressor 69

2.10.7. Massa total de refrigerante no supermercado 69

2.11. Cálculo de TEWI (Total Equivalent Warming Impact) 69

2.12. Análise LCCP (Life Cycle Climate Performance) 70

3. Método de solução 72

3.1. Introdução 72

3.2. Dados de entrada 73

3.2.1. Dados de entrada gerais no programa 73

3.2.2. Dados característicos dos componentes do sistema 74

3.3. Plataforma computacional 75

3.4. Desenvolvimento do código computacional 76

3.5. Procedimento computacional 76 
3.5.1. Algoritmo geral do ciclo termodinâmico para cada condição de operação

3.5.2. Algoritmo específico para misturas não azeotrópicas

3.5.2.1. Algoritmo para o condensador

3.5.2.2. Algoritmo para o Evaporador

4. Validação do modelo

4.1. Introdução

4.2. Validação do modelo desenvolvido

4.2.1. Comparação com o trabalho teórico de Kazachki (2007)

Simulação do consumo energético dos compressores de um supermercado

4.2.2. Comparação como o trabalho experimental de Sawalha (2008) Simulação do COP de sistema cascata operando com amônia e $\mathrm{CO}_{2} \quad 95$ 4.2.3. Comparação com trabalho teórico da Arthur D. Little (2002) Simulação do consumo energético global e do LCCP de um supermercado

5. Resultados 102

5.1. Sistema de expansão direta 102

5.2. Sistema com fluido secundário 110

5.3. Sistema Cascata 112

5.4. Comparação dos sistemas (DX, FS e Cascata)

6. Conclusões e recomendações para trabalhos futuros 121

6.1. Conclusões 121

6.2. Sugestões para trabalhos futuros 


\section{Lista de tabelas}

Tabela 1 - Requerimentos de temperaturas para diferentes produtos (Baxter et al., 2004)

Tabela 2 - Cargas de refrigeração específicas para diferentes expositores frigoríficos (Henderson e Khattar, 1996) 47

Tabela 3 - Diagrama de Moody (Fischer, 2003)

Tabela 4 - Valores de K para acessórios com junção aparafusada (ASHRAE, 1985)

Tabela 5 - Valores de K para acessórios com junção de flange soldado (ASHRAE, 1985)

Tabela 6 - Dada geral da simulação (Kazachki, 2007)

Tabela 7 - Dados específicos do sistema de refrigeração do

supermercado

(Kazachki, 2007)

Tabela 8 - Análise BIN do trabalho de Kazachki (2007) para expansão direta

Tabela 9 - Resultados obtidos com o modelo para expansão direta

Tabela 10 - Dados gerais da simulação

Tabela 11 - Dados específicos do sistema de refrigeração do

supermercado

(Kazachki, 2007)

Tabela 12 - Análise BIN do trabalho do Kazachki (2007) para fluido secundário

Tabela 13 - Análise BIN do trabalho do presente modelo para fluido secundário

Tabela 14 - Parâmetros básicos de funcionamento do sistema cascata 96

Tabela 15 - Resultados globais da simulação

Tabela 16 - Resultados do COP do presente modelo

Tabela 17 - Tabela comparativa entre os resultados de Sawala (2008) e o presente modelo 
Tabela 19 - Dados específicos do sistema de refrigeração do supermercado

Tabela 20 - Consumo Energético anual, TEWI e o LCCP, previstos para o presente modelo (expansão direta)

Tabela 21 - Consumo Energético anual, TEWI e o LCCP, previstos para o presente modelo (fluido secundario)

Tabela 22 - Tabela comparativa entre os resultados de

Arthur D. Little (2002) e o presente modelo 100

Tabela 23 - Dados gerais de locação e do sistema a ser utilizado 102

Tabela 24 - Condições de operação do sistema 103

Tabela 25 - Consumo energético e LCCP para R404A -

Expansão direta

103

Tabela 26 - Consumo energético e LCCP para R407A -

Expansão direta

105

Tabela 27 - Consumo energético e LCCP para R407C -

Expansão direta 106

Tabela 28 - Consumo energético e LCCP para o R407F 107

Tabela 29 - Comparação entre os diferentes refrigerantes -

Expansão direta 109

Tabela 30 - Consumo energético e LCCP para o R407F -

Fluido secundário

Tabela 31 - Parâmetros de funcionamento do sistema em cascata 113

Tabela 32 - Consumo energético e LCCP para a Cascata $\left(\mathrm{R} 407 \mathrm{~F} / \mathrm{CO}_{2}\right)$

Tabela 33 - Consumo energético e LCCP para o sistema em Cascata 115 (R407F/R410A)

Tabela 34 - Tabela comparativa para o sistema cascata 


\section{Lista de figuras}

Figura 1 - Percentual de consumo energético da refrigeração respectivo a outros sistemas (Panesi, 2008) 20

Figura 2 - Planta da disposição de expositores em um supermercado (Baxter et al.,2004) 21

Figura 3 - Tipos de expositores frigoríficos (Baxter et al., 2004) 23

Figura 4 - Corte transversal de um expositor multi-plataforma (Baxter et al., 2004)

Figura 5 - Percentual de consumo energético da refrigeração em um supermercado (Baxter et al., 2004)

Figura 6 - Sistema de expansão direta para baixa e média temperatura

Figura 7 - Diagrama p-h para o ciclo de refrigeração de expansão direta - Baixa temperatura.

Figura 8 - Diagrama p-h para o ciclo de refrigeração de expansão direta - Média temperatura.

Figura 9 - Sistema de fluido secundário para baixa e média temperatura

Figura 10 - Diagrama p-h para o ciclo de refrigeração de expansão direta trabalhando com fluido secundário - Baixa temperatura.

Figura 11 - Diagrama p-h para o ciclo de refrigeração de expansão direta trabalhando com fluido secundário - Média temperatura.

Figura 12 - Sistema de cascata de alta e baixa temperatura

Figura 13 - Diagrama p-h para os ciclos em cascata

Figura 14 - Diagrama P-h do ciclo térmodinamico operando com R404A (exemplo)

Figura 15 - Distribuição das tubulações na montante e na jusante do evaporador

Figura 16 - Distribuição das tubulações na montante e na jusante do condensador

Figura 17 - Carga de refrigerante em trocadores de calor 
Figura 18 - Geometria do tanque vertical 67

Figura 19 - Geometria do tanque horizontal 68

Figura 20 - Diagrama P-h (a) para um refrigerante puro ou mistura azeotrópica e (b) para uma mistura não-azeotrópica 77

Figura 21 - Diagrama Erro vs. Temperatura do líquido saturado 84

Figura 22 - Ciclo de refrigeração mostrando as pressões de condensação e de evaporação

Figura 23 - Comparação entre o presente modelo e o de Kazachki kWh/ano (2007) 92

Figura 24 - Figura comparativa entre ambas as opções - kWh/ano 95

Figura 25 - Gráficos de barras do consumo energético R404A -

Expansão direta

Figura 26 - Gráfico de barras para LCCP R404A - Expansão direta 104

Figura 27 - Gráfico de barras do consumo energético R407A -

Expansão direta

105

Figura 28 - Gráfico de barras para LCCP R407A - Expansão direta 105

Figura 29 - Gráfico de barras do consumo energético R407C -

Expansão direta

106

Figura 30 - Gráfico de barras para LCCP R407C - Expansão direta

Figura 31 - Gráfico de barras do consumo energético R407F _

Expansão direta

108

Figura 32 - Gráfico de barras para LCCP R407F - Expansão direta

Figura 33 - Gráfico de comparação entre alternativas (consumo de energia)

Figura 34 - Gráfico de comparação entre alternativas (LCCP)

Figura 35 - Gráfico de barras do consumo energético com R407F _

Fluido secundário

Figura 36 - Gráfico de barras para LCCP com R407F _

Fluido secundário

Figura 37 - Gráfico de barras do consumo energético a Cascata

Figura 38 - Gráfico de barras para LCCP no sistema em Cascata $\left(\mathrm{R} 407 \mathrm{~F} / \mathrm{CO}_{2}\right)$ - Sistema de baixa Temperatura 
Figura 39 - Gráfico de barras para LCCP no sistema em Cascata (R407F/CO2) - Sistema de alta Temperatura

Figura 40 - Gráfico de barras do consumo energético - Sistemas em cascata

(R407F/R410A)

Figura 41 - Gráfico de barras para LCCP a Cascata (R407F/410A) Baixa Temperatura

Figura 42 - Gráfico de barras para LCCP a Cascata (R407F/R410A) -

Alta Temperatura

Figura 43 - Gráfico de barras para o consumo energético

(Circuito em cascata)

Figura 44 - Gráfico comparativo de barras para do LCCP do sistema em cascata

Figura 45 - Gráfico de barras da comparação entre as alternativas (Consumo energético)

Figura 46 - Gráfico de barras da comparação entre as alternativas (LCCP) 


\section{Lista de símbolos}

\begin{tabular}{|c|c|c|c|}
\hline & $a$ & Eficiência radiativa & {$\left[\mathrm{Wm}^{-2} \mathrm{~kg}^{-1}\right]$} \\
\hline & $A$ & Área & {$\left[\mathrm{m}^{2}\right]$} \\
\hline & $c_{p}$ & Calor específico a pressão constante & {$[-]$} \\
\hline & COP & Coeficiente de desempenho & {$[-]$} \\
\hline & $D$ & Diâmetro & [m] \\
\hline & $d t$ & Diferença de tempo & [anos] \\
\hline & $F C$ & Fator de carga & {$[-]$} \\
\hline & $E D$ & Efeito Direto & {$\left[\mathrm{kg}_{\mathrm{CO} 2}\right]$} \\
\hline & $E$ & Consumo de energia & {$[\mathrm{kWh}]$} \\
\hline & EI & Efeito Indireto & {$\left[\mathrm{kg}_{\mathrm{CO} 2}\right]$} \\
\hline & Erro & Erro incorrido & {$[-]$} \\
\hline & Fluido & Mistura azeotrópica ou não-azeotrópica & {$[-]$} \\
\hline & $F M$ & Fração de massa & {$[-]$} \\
\hline & $F R$ & Emissão de $\mathrm{CO}_{2}$ equiv. na fabricação dc & {$\left[\mathrm{kg}_{\mathrm{CO} 2} \mathrm{~kg}_{\mathrm{Ref}}{ }^{-1}\right]$} \\
\hline & $g$ & Aceleração da gravidade & {$\left[\mathrm{m} \mathrm{s}^{-2}\right]$} \\
\hline & $G W P$ & Global Warming Potential & {$\left[\mathrm{kg}_{\mathrm{CO} 2} \mathrm{~kg}_{\mathrm{Ref}^{-1}}\right]$} \\
\hline$\frac{N}{\infty}$ & $h$ & Entalpia específica & {$\left[\mathrm{kJ} \mathrm{kg}^{-1}\right]$} \\
\hline o & $H$ & Altura ou nível do refrigerante & {$[\mathrm{m}]$} \\
\hline$\frac{z}{\pi}$ & Hman & Altura de pressão da bomba & [m] \\
\hline 8 & Incpt & Intercepto com o eixo vertical & {$[-]$} \\
\hline a & $K$ & Constante do acessório & {$[-]$} \\
\hline & $L$ & Comprimento & [m] \\
\hline 艼 & $L C C P$ & Life Cycle Climate Performance & {$\left[\mathrm{kg}_{\mathrm{CO} 2}\right]$} \\
\hline 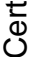 & $m$ & Massa & [kg] \\
\hline & $\dot{m}$ & Vazão de massa & {$\left[\mathrm{kg} \mathrm{s}^{-1}\right]$} \\
\hline 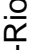 & $N C$ & Número de cilindros & {$[-]$} \\
\hline دُ & NH & Número de Horas & [h] \\
\hline & $P$ & Pressão & {$[\mathrm{kPa}]$} \\
\hline & $q$ & Fluxo de calor específico & {$\left[\mathrm{kJ} \mathrm{kg}^{-1}\right]$} \\
\hline & $\dot{Q}$ & Taxa de transferência de calor & {$[\mathrm{kW}]$} \\
\hline & $q$ & Taxa de transferência de calor por área & {$\left[\mathrm{kW} \mathrm{m}^{-2}\right]$} \\
\hline & $r(t)$ & Função do tempo de vida do gás traço & {$[-]$} \\
\hline & $R$ & Raio & [m] \\
\hline & $C R$ & Conversão regional de energia & {$\left[\mathrm{kg}_{\mathrm{co} 2} \mathrm{~kW}^{-1}\right]$} \\
\hline & $R C R$ & Razão de carga de refrigerante & {$[-]$} \\
\hline & $R D$ & Razão de densidades líquido/vapor & {$[-]$} \\
\hline & $R e$ & Número de Reynolds & {$[-]$} \\
\hline & $s$ & Entropia específica & {$\left[\mathrm{KJ} \mathrm{kg}^{-1} \mathrm{~K}^{-1}\right]$} \\
\hline & $S$ & Secção transversal & {$\left[\mathrm{m}^{2}\right]$} \\
\hline & $S A$ & Grau de superaquecimento & {$\left[{ }^{\circ} \mathrm{C}\right]$} \\
\hline & $\operatorname{slp}$ & Pendente & {$[-]$} \\
\hline & $S M$ & Grau de sub-resfriamento mecânico & {$\left[{ }^{\circ} \mathrm{C}\right]$} \\
\hline & $t$ & Tempo & [anos] \\
\hline & $T$ & Temperatura & {$\left[{ }^{\circ} \mathrm{C}\right]$} \\
\hline & Taxa & Taxa de rejeição de calor & [kW] \\
\hline
\end{tabular}




$\begin{array}{lll}T E W I & \text { Total Equivalent Warming Impact } & {\left[\mathrm{kg}_{\mathrm{co} 2}\right]} \\ T H & \text { Horizonte temporal } & {[\mathrm{anos}]} \\ T H R & \text { Rejeição total de calor no condensador } & {[\mathrm{kW}]} \\ T V & \text { Tempo de vida } & {[\mathrm{anos}]} \\ V & \text { Velocidade } & {\left[\mathrm{m} \mathrm{s}^{-1}\right]} \\ \dot{V} & \text { Deslocamento volumétrico } & {\left[\mathrm{m}^{3} \mathrm{~s}^{-1}\right]} \\ \dot{W} & \text { Potência } & {[\mathrm{kW}]} \\ x & \text { Gás refrigerante analisado } & {[-]} \\ x(t) & \text { Função do tempo de vida da substância } & {[-]} \\ X & \text { Título } & {[-]} \\ Z & \text { Altitude } & {[\mathrm{m}]}\end{array}$

Símbolos gregos

$\beta \quad$ Cte. para o cálculo da massa de refrigerante[\%]

$\delta \quad$ Fração de fluxo de calor [\%]

$\Delta P \quad$ Diferença de pressão $\quad[\mathrm{kPa}]$

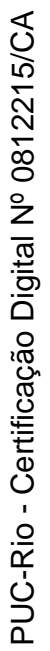

$\begin{array}{lll}\Delta T & \text { Diferença de temperatura } & \left.{ }^{\circ} \mathrm{C}\right]\end{array}$

$\eta \quad$ Eficiência

$f \quad$ Fator de atrito

$\rho \quad$ Densidade $\quad\left[\mathrm{kg} \mathrm{m}^{-3}\right]$

$\varnothing \quad$ Porcentagem de vazamento [\%]

$\vartheta \quad$ Viscosidade dinâmica $\quad\left[\mathrm{kg} \mathrm{m}^{-1} \mathrm{~s}^{-1}\right]$

Subscritos

$\begin{array}{ll}b & \text { Relativo à bomba } \\ \text { bal } & \text { Expositor frigorifico } \\ \text { Bolha } & \text { Ponto de bolha } \\ B T & \text { Baixa temperatura } \\ C D & \text { Condensador } \\ \text { Comp } & \text { Compressor } \\ \text { Cond, COND } & \text { Condensador ou condensação } \\ \text { des } & \text { Descarga } \\ e & \text { Entrada } \\ E Q & \text { Condição equivalente } \\ E S & \text { Específico } \\ \text { Evap, EVAP } & \text { Evaporador ou evaporação } \\ f & \text { Fluido } \\ G & \text { Global } \\ \text { glide } & \text { Temperatura de glide } \\ \text { IHX } & \text { Trocador de calor intermediário. } \\ \text { IN } & \text { Entrada } \\ \text { ini } & \text { Inicial } \\ \text { iso } & \text { Isentrópica } \\ \text { liq } & \text { Líquido } \\ \text { linhas } & \text { Linha de liquido ou vapor }\end{array}$




$\begin{array}{ll}\text { man } & \text { Manifold ou distribuidor } \\ M E & \text { Temperatura ótima de evaporação } \\ M E D & \text { Média } \\ M C & \text { Temperatura ótima de condensação } \\ M T & \text { Meia Temperatura } \\ \text { novo } & \text { Variável nova } \\ \text { Orvalho } & \text { Ponto de orvalho } \\ \text { OUT } & \text { Saída } \\ P & \text { Pistão } \\ r & \text { Relativo ao gás traço }\left(\mathrm{CO}_{2}\right) \\ R & \text { Condição real } \\ R E F & \text { Refrigerante } \\ R E J & \text { Rejeitado } \\ S & \text { Saída } \\ S M & \text { Sub-resfriamento mecânico } \\ S R & \text { Taxa de sub-resfriamento } \\ \text { suc } & \text { Sucção } \\ S U P & \text { Referente ao supermercado } \\ t & \text { De trabalho } \\ t c & \text { De trabalho crítico } \\ \text { total } & \text { Total de alguma soma aritmética } \\ T r & \text { Tanque Reservatório } \\ \text { tub } & \text { Tubulação } \\ \text { val, valv } & \text { Válvula } \\ v . c . & \text { Volume de controle } \\ \text { vap } & \text { Vapor } \\ \text { vaz } & \text { Vazamento } \\ x & \text { Relativo a gás refrigerante analisado } \\ & \\ & \end{array}$

